\title{
Subject Death Flag
}

National Cancer Institute

\section{Source}

National Cancer Institute. Subject Death Flag. NCI Thesaurus. Code C117451.

An indication of whether the subject has died. 